\title{
Determination of vertebral range of motion using inertial measurement units in 27 Franches-Montagnes stallions and comparison between conditions and with a mixed population
}

\author{
C. HEIM*, T. PFAU ${ }^{\dagger}$, V. GERBER, C. SCHWEIZER, M. DOHERR*, G. SCHÜPBACH-REGULA* and S. WITTE \\ Swiss Institute of Equine Medicine, Vetsuisse Faculty, University of Berne and ALP-Haras, Berne, Switzerland \\ 'Department of Clinical Science and Services, The Royal Veterinary College, University of London, London, UK \\ tVeterinary Public Health Institute, Vetsuisse Faculty, University of Berne, Berne, Switzerland. \\ *Correspondence email: corsin.heim@bluewin.ch; Received: 06.05.14; Accepted: 19.04.15
}

\begin{abstract}
Summary
Reasons for performing study: The diagnosis of equine back disorders is challenging. Objectively determining movement of the vertebral column may therefore be of value in a clinical setting.

Objectives: To establish whether surface-mounted inertial measurement units (IMUs) can be used to establish normal values for range of motion (ROM) of the vertebral column in a uniform population of horses trotting under different conditions.

Study design: Vertebral ROM was established in Franches-Montagnes stallions and a general population of horses and the variability in measurements compared between the two groups. Repeatability and the influence of specific exercise condition (on ROM) were assessed. Finally, attempts were made to explain the findings of the study through the evaluation of factors that might influence ROM.

Methods: Dorsoventral (DV) and mediolateral (ML) vertebral ROM was measured at a trot under different exercise conditions in 27 FranchesMontagnes stallions and six general population horses using IMUs distributed over the vertebral column.

Results: Variability in the ROM measurements was significantly higher for general population horses than for Franches-Montagnes stallions (both DV and ML ROM). Repeatability was strong to very strong for DV measurements and moderate for ML measurements. Trotting under saddle significantly reduced the ROM, with sitting trot resulting in a significantly lower ROM than rising trot. Age is unlikely to explain the low variability in vertebral ROM recorded in the Franches-Montagnes horses, while this may be associated with conformational factors.

Conclusions: It was possible to establish a normal vertebral ROM for a group of Franches-Montagnes stallions. While within-breed variation was low in this population, further studies are necessary to determine variation in vertebral ROM for other breeds and to assess their utility for diagnosis of equine back disorders.
\end{abstract}

Keywords: horse; back; vertebral column; objective; range of motion; inertial measurement unit

\section{Introduction}

Objective measurements of head and trunk symmetry as an adjunct to subjective lameness evaluation are now routinely performed in a field setting using small surface-mounted inertial measurement units (IMUs) [1-3]. The inability to directly assess the three-dimensional range of motion (ROM) of the vertebral column and the lack of reliability in subjectively detecting movement asymmetries below a threshold level of 25\% [4] limits the value of the information that can be obtained during clinical evaluation of horses with suspected back pain. A recent study validated IMUs against motion capture for determining thoracolumbar ROM and showed acceptable accuracy and good levels of consistency [5].

The purpose of the current study was to establish whether surfacemounted IMUs can be used to establish normal values for ROM of the vertebral column in a uniform population of horses trotting under different conditions. For the purpose of this study Franches-Montagne horses were used. This light cold-blood breed, indigenous to Switzerland, is represented in most equestrian disciplines.

Our specific hypotheses were as follows:

- Variability of ROM measurements (total dorsoventral (DV) motion measured in millimetres and total mediolateral (ML) motion measured in millimetres) at the same sensor locations between horses and within a condition is significantly higher in the general horse population than in a group of Franches-Montagnes stallions, trotted in-hand (without a saddle).

- Repeatability of objectively measured vertebral ROM in 10 FranchesMontagnes stallions without clinical signs of back pain and trotted in-hand is high.

- Changing the condition under which the Franches-Montagnes stallions are measured results in systematic changes in ROM.
Age, rump length, height at withers and the ratio of rump length to height at withers (RL:HW) were evaluated as factors potentially influencing or explaining our findings.

\section{Material and methods}

\section{Horses}

For inclusion horses had to be free of any clinical evidence of back pain, pass a subjective lameness evaluation and have an objectively measured symmetry index that fell within a previously reported normal range $[2,6]$. These evaluations were performed solely for the purpose of this study. After the exclusion of 11 Franches-Montagnes horses and one general population horse that did not meet these criteria, the Franches-Montagnes group consisted of 27 stallions (age range: 4-21 years, height range: 151-162 cm, rump length range: 150-166 $\mathrm{cm}$ ); the general population consisted of six horses (one stallion, four geldings, one mare; breeds: Lusitano, Swiss Warmblood, age range: 5-13 years, height range: 160-176 cm, rump length range: 154-179 cm). Horses were instrumented and measured at the Swiss National Stud. All horses were in daily exercise at the time they were measured. While the Franches-Montagnes stallions were exercised in a 3-day cycle ( 1 day ridden, the second driven and the third exercised on the horsewalker), the general population horses were used for dressage, in daily exercise or competition.

\section{Equipment}

Five IMUs $(\mathrm{MTX})^{\mathrm{a}}$ were attached to a wireless transmitter (XBus) ${ }^{\mathrm{a}}$ via serial connections and then attached to the horses with adhesive dressing ${ }^{b}$ at the following landmarks: poll (1), summit of the dorsal spinous process of 
the 12th thoracic vertebrae (2) and 3rd lumbar vertebrae (3), between the tuber sacrale (4) and summit of the dorsal spinous process of the 3rd sacral vertebrae (5) (Fig 1). These landmarks were identified by a qualified chiropractor.

Each IMU consists of a 3-axis accelerometer (full scale $\pm 180 \mathrm{~m} / \mathrm{s}^{2}$ ), a 3-axis gyroscope (full scale $\pm 1200 \%$ s) and a 3-axis magnetometer (full scale \pm 750 mGauss) resulting in a static accuracy of $<0.5^{\circ}$ for roll and pitch and $<1^{\circ}$ for heading measurements. With a rider in place IMU 2 was discarded. Data were transmitted to a laptop computer at a sampling rate of $50 \mathrm{~Hz}$ running MT Manager software ${ }^{a}$ and processed using custom software ${ }^{\mathrm{c}}$

\section{Data collection}

Each horse was given an initial ridden warm-up of 10-15 min prior to being instrumented and measured. Horses were trotted over a distance of $50 \mathrm{~m}$ (repeated until sufficient strides (at least 25) of synchronised data were recorded). Exercise conditions under which ROM was measured were as follows:

- Trotting in-hand without saddle.

- Trotting in-hand with saddle.

- Rising trot under saddle.

- Sitting trot under saddle.

All horses were ridden by the same experienced rider and measurements made in the same sand-based arena. To assess repeatability 10 horses underwent a repeat measurement approximately 1 month following acquisition of the first set of data.

\section{Data analysis}

A standard right-handed orthogonal Cartesian coordinate system was used for orientation (craniocaudal or $x$ : positive axis directed along the line of progression; DV or z: axis vertical [aligned with gravitational field] and positive in the upward direction; ML or $y$ : axis perpendicular to the first two axes). Craniocaudal ( $x$ ), ML (y) and DV (z) displacement data in the horse based reference system were calculated following published methods $[7,8]$ with modified highpass filter frequencies chosen as $1.5 \mathrm{~Hz}$ for DV and $0.75 \mathrm{~Hz}$ for lateral movement. Range of motion was calculated for each stride [9] as the difference between the maximum and the minimum value measured within each stride. The median ROM value for each horse under each exercise condition was then used for statistical analysis.

Statistical analysis was carried out in NCSS ${ }^{d}$ and data were considered significant for $\mathrm{P}<0.05$. Normality of the data was tested using the ShapiroWilk test. For all means, 95\% lower and upper confidence limits (LCL and UCL) were calculated with the formula mean $\pm(1.96 \times$ standard error of the mean). Similarly, LCL and UCL were calculated for the standard deviations. Hypothesis 1 was tested by comparing 95\% lower and upper confidence intervals for the standard deviation between the FranchesMontagnes and general population groups. The Modified Levene Equal Variance Test was used to test the differences in variance within the groups for statistical significance. Repeatability of the ROM measurements (Hypothesis 2) was assessed by the Pearson correlation coefficient of the two measures within the same horse. In order to assess the effect of changing conditions on ROM (Hypothesis 3), data were analysed using a repeated measures ANOVA. A mixed model which allows simultaneous inclusion of categorical and continuous predictor variables was used for

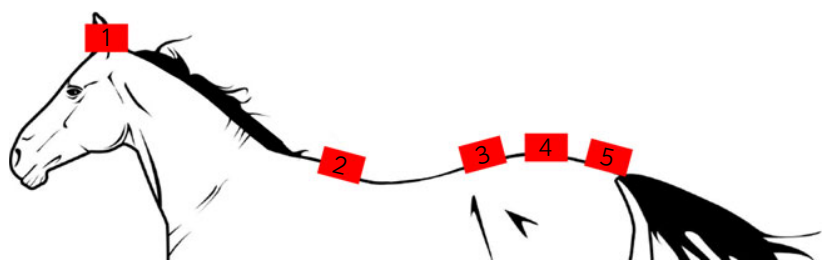

Fig 1: Placement of inertial measurement units along the spine: poll (1), summit of the dorsal spinous process of the 12th thoracic vertebrae (2) and 3rd lumbar vertebrae (3), tuber sacrale (4) and summit of the dorsal spinous process of the 3rd sacral vertebrae (5) this analysis. In case of significance, a pair-wise comparison of the different conditions was performed ( $P$ values Bonferroni corrected). Stride frequency as a proxy for trotting speed [10-12] was included in the mixed model, trotting speed affecting most movement features even in sound horses [12] and therefore being a main factor of the motion.

The biomechanical variables age, rump length (distance from shoulder joint to tuber ischiadicum), height at withers and RL:HW were determined and the size of the standard deviation and its confidence intervals compared between Franches-Montagnes stallions and general population horses. The Modified Levene Equal Variance Test was used to assess the statistical significance of different variances. Additionally, the biomechanical variable age was analysed by dividing the study population (FranchesMontagnes and general population horses) into age groups (2, 3 or 4 groups) and using a repeated measures ANOVA to investigate interactions between age and DV and ML ROM

\section{Results}

\section{Hypothesis 1}

Dorsoventral and ML vertebral ROM is shown in Figures 2 and 3 for Franches-Montagnes stallions and the general population. Absolute measurements can be found in the supporting information (Supplementary Items 1 and 2). The variance of the ROM measurements of FranchesMontages stallions and general population horses confirmed a significantly larger variance in general population ROM measurements (DV: $\mathrm{P}<0.02, \mathrm{ML}$ : $\mathrm{P}<0.001$ ).

\section{Hypothesis 2}

The results of the Pearson correlation, reflecting degree of repeatability of the measurements made at each of the IMU landmarks under each of the conditions are listed in Table 1.

In general a strong to very strong repeatability was noted with regard to DV ROM, irrespective of exercise condition. Mediolateral ROM was less repeatable, ranging from a weak to a very strong correlation.

\section{Hypothesis 3}

Changes in DV and ML ROM that occurred at the different IMU locations in the Franches-Montagnes stallions when changing condition are shown in Table 2 and Figures 4 and 5. For IMU 2 (summit of the dorsal spinous process of the 12th thoracic vertebrae), comparison was not always possible as this location was lost under the saddle.

No significant difference was noted in DV ROM when comparing horses trotted in-hand with and without a saddle alone. Mean DV ROM was significantly less when trotting under saddle compared with when trotting in-hand. DV ROM was significantly less during sitting trot when compared with rising trot. Stride frequency was significantly associated with increased DV movement in the ANOVA model $(P<0.001)$. The interaction term between exercise condition and stride frequency was not significant, and the effect of exercise condition on DV ROM remained unchanged after correction for stride frequency in a multivariate ANOVA model.

No significant difference was noted in ML ROM when comparing horses trotted in-hand with and without a saddle alone. Trotting in-hand conditions differ significantly from trot under saddle conditions. While there is significantly greater ML ROM at IMU 1 (poll) under saddle, there is a trend towards less ML ROM at the more caudal locations, some of these differences being significant. Significantly less lateral ROM was noted at both IMU 1 (poll) and IMU 3 (summit of the dorsal spinous process of the 3rd lumbar vertebrae) when horses were ridden in rising trot, when compared with sitting trot. As noted with DV ROM, controlling for stride frequency in a multivariable model did not alter the effect of exercise condition on ML ROM.

\section{Factors}

There was no significant difference in variance between age of FranchesMontagnes stallions and general population horses; however, all other 

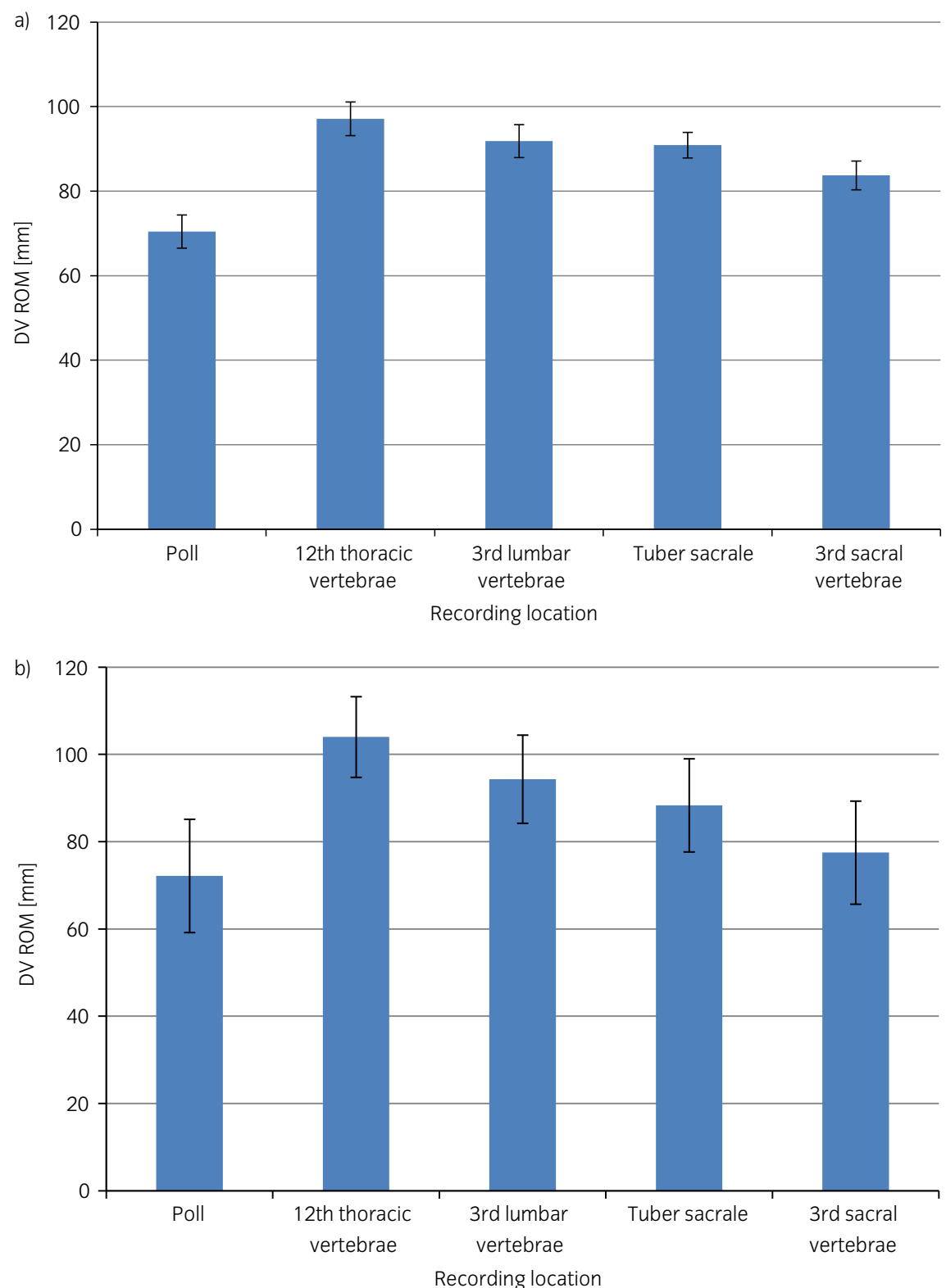

Fig 2: Mean dorsoventral range of motion (DV ROM; in $\mathrm{mm}$ ) of the vertebral column at the five locations along the spine shown in Figure 1 recorded in 27 FranchesMontagnes stallions (a), and six general purpose horses (b). Error bars represent 95\% lower and upper confidence limits of the mean.

factors associated with conformation (rump length, height at withers and the ratio of rump length to height at withers [RL:HW]) differed significantly in their variance and are therefore potential candidate factors associated with the difference in ROM variance between Franches-Montagnes stallions and general population horses (Table 3).

The results of the repeated measures ANOVA can be found in the supporting information (Supplementary Items 3-5). Only one significant influence on DV ROM was found when horses were split into 2 groups according to age, and exercised at rising trot under saddle (condition 3 ). All other comparisons were not significant.

\section{Discussion}

In horses trotted in-hand IMU 1 (poll) measured least DV ROM of all IMUs. In the sound horses included in this study, this finding may support a stabilising role of a stationary head for the remaining vertebral column, with larger movements of this lever arm only occurring when redistribution of the centre of mass is necessary, such as in the case of lameness [4]. In agreement with numerous in vitro and in vivo studies, our study observes a gradual decrease in DV ROM from cranial to caudal [13-17]. Our data are also comparable with those obtained from preliminary studies using IMUs applied dorsal to the vertebrae in ponies [5]. Absolute DV ROM data are, however, consistently higher in both groups studied here.

Mediolateral ROM is less consistent than DV ROM. A possible explanation for this apparent greater variation may be the 'restrictive' influence of gravity in a DV direction compared with the unrestricted freedom to move laterally. Restricting lateral movement (e.g. by imposing the centripetal forces of a circle) may be required to make ML ROM a more consistent measure. However, other confounding factors such as circle radius and speed should be taken into consideration $[18,19]$. Mediolateral output data from surface-mounted IMUs must also be interpreted with caution. Given the discrepancy between the movement of the IMU 


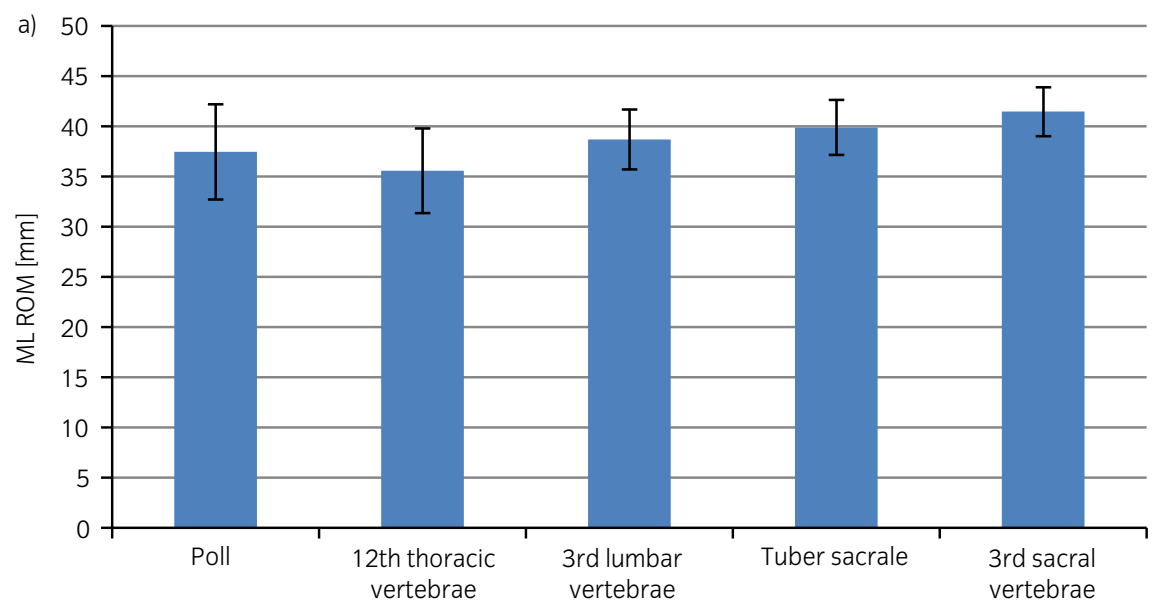

Recording location

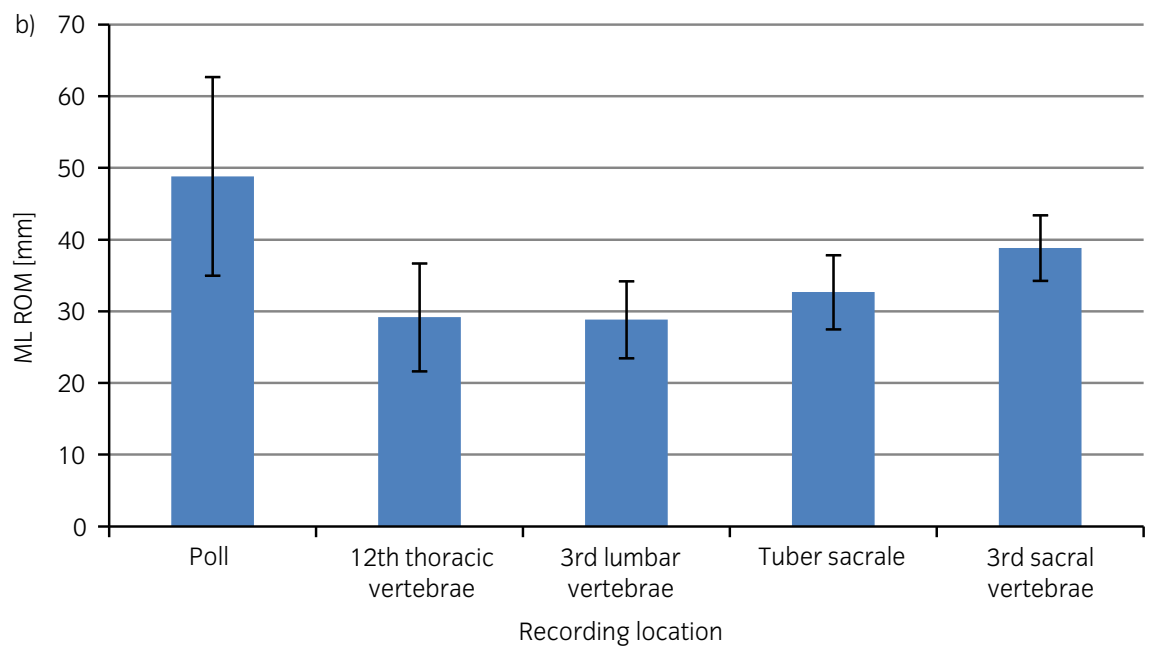

Fig 3: Mean mediolateral range of motion (ML ROM; in $\mathrm{mm}$ ) of the vertebral column at the five locations along the spine shown in Figure 1 recorded in 27 FranchesMontagnes stallions (a) and six general purpose horses (b). Error bars represent $95 \%$ lower and upper confidence limits of the mean.

TABLE 1: Pearson correlations between data on dorsoventral (DV) and mediolateral (ML) range of motion (ROM) of the vertebral column recorded on two occasions at the five locations along the spine, shown in Figure 1, from 10 Franches-Montagnes stallions exercised under four conditions

\begin{tabular}{lll}
\hline Exercise condition & DV ROM & ML ROM \\
\hline Trotting in-hand without saddle & 0.74 & 0.38 \\
Trotting in-hand with saddle & 0.84 & 0.35 \\
Rising trot under saddle & 0.94 & 0.79 \\
Sitting trot under saddle & 0.94 & 0.95 \\
Overall & 0.88 & 0.68 \\
\hline
\end{tabular}

attached to the skin and the underlying bony structure (due to skin mobility), these units are unable to differentiate between true lateral bending and axial rotation of the vertebral column [20]. This so-called skin displacement artefact is a source of error well recognised in human and equine gait analysis [21-23].

Mediolateral ROM was observed to increase caudally. Although this finding is consistent with a single study using IMUs [5], it is in contrast to some previous studies $[13,14,16]$. Data for the latter studies were generated on a treadmill and recorded using motion capture techniques.
Consistent with Gomez Alvarez et al. [24], the authors speculate that restriction in lateral ROM on the treadmill may explain the difference between studies.

Variability of both the DV and ML ROM measurements was significantly higher for general population horses than for Franches-Montagnes stallions. Our first hypothesis can therefore be upheld. In the 10 horses that were measured a second time the overall Pearson correlation value for each exercise condition indicated a strong to very strong repeatability for the DV ROM measurements. Mediolateral measurements are less reliable with only moderate repeatability. Possible explanations for these findings are discussed above.

The systematic changes in mean ROM measurements seen with changes in exercise condition are summarised in Table 2. The finding that the saddle alone has no significant influence on either DV or ML ROM is consistent with previous studies using motion capture techniques [17]. In the current study, the presence of a rider was associated with a significant decrease in DV ROM at almost all IMU locations and a decrease in ML ROM at some IMU locations when compared with the conditions without a rider. The weight of the rider has a direct biomechanical effect on the musculoskeletal system of the horse [25-27], the magnitude of which is directly associated with the rider's body weight. The bow and string model dictates that a weight exerted on the horse's thoracolumbar spine will result in its extension [28]. This has been confirmed using a dead weight [17]. Additional kinematic studies have shown variable results ranging from 
TABLE 2: Repeated measures ANOVA of differences in dorsoventral and mediolateral range of vertebral movement recorded at five spinal locations under four exercise conditions in Franches-Montagnes stallions

\begin{tabular}{|c|c|c|c|c|c|c|c|c|c|c|c|}
\hline & & \multicolumn{2}{|l|}{ Poll } & \multicolumn{2}{|c|}{$\begin{array}{l}\text { 12th thoracic } \\
\text { vertebrae }\end{array}$} & \multicolumn{2}{|c|}{$\begin{array}{l}\text { 3rd lumbar } \\
\text { vertebrae }\end{array}$} & \multicolumn{2}{|c|}{ Tuber sacrale } & \multicolumn{2}{|c|}{$\begin{array}{l}\text { 3rd sacral } \\
\text { vertebrae }\end{array}$} \\
\hline & & Mean & $\neq$ & Mean & $\neq$ & Mean & $\neq$ & Mean & $\neq$ & Mean & $\neq$ \\
\hline \multicolumn{12}{|c|}{ DV ROM } \\
\hline 1 & Trotting in-hand without saddle & 70.44 & 3 & 97.12 & $\star$ & 91.84 & 3,4 & 90.88 & 3,4 & 83.72 & 4 \\
\hline 2 & Trotting in-hand with saddle & 69.74 & 3 & 96.96 & * & 91.48 & 3,4 & 90.30 & 3,4 & 82.44 & 4 \\
\hline 3 & Rising trot under saddle & 65.19 & 1,2 & 111.67 & * & 86.07 & $1,2,4$ & 86.52 & $1,2,4$ & 81.19 & 4 \\
\hline 4 & $\begin{array}{l}\text { Sitting trot under saddle } \\
P \text { value }\end{array}$ & $\begin{array}{l}68.23 \\
<0.001\end{array}$ & & $\begin{array}{l}100.46 \\
*\end{array}$ & * & $\begin{array}{l}77.19 \\
<0.001\end{array}$ & $1,2,3$ & $\begin{array}{l}77.62 \\
<0.001\end{array}$ & $1,2,3$ & $\begin{array}{l}76.54 \\
<0.001\end{array}$ & $1,2,3$ \\
\hline \multicolumn{12}{|c|}{ ML ROM } \\
\hline 1 & Trotting in-hand without saddle & 37.44 & 3,4 & 35.56 & * & 38.78 & 3 & 40.04 & 3 & 42.67 & 4 \\
\hline 2 & Trotting in-hand with saddle & 34.19 & 3,4 & 44.00 & * & 38.68 & 3 & 39.88 & 3 & 41.44 & \\
\hline 3 & Rising trot under saddle & 52.63 & $1,2,3$ & 31.22 & * & 32.89 & $1,2,4$ & 35.19 & 1,2 & 39.67 & \\
\hline 4 & $\begin{array}{l}\text { Sitting trot under saddle } \\
\mathrm{P} \text { value }\end{array}$ & $\begin{array}{l}66.42 \\
<0.001\end{array}$ & $1,2,4$ & $\begin{array}{l}30.77 \\
\star\end{array}$ & * & $\begin{array}{l}36.08 \\
<0.001\end{array}$ & 3 & $\begin{array}{l}36.65 \\
<0.002\end{array}$ & & $\begin{array}{l}38.62 \\
<0.04\end{array}$ & 1 \\
\hline
\end{tabular}

DV, dorsoventral; ML, mediolateral; ROM, range of vertebral motion. Recording locations are shown in Figure 1. Column $\neq$ lists exercise condition(s) in which a difference was found. *no comparison possible as data recorded from this location were inconsistent.

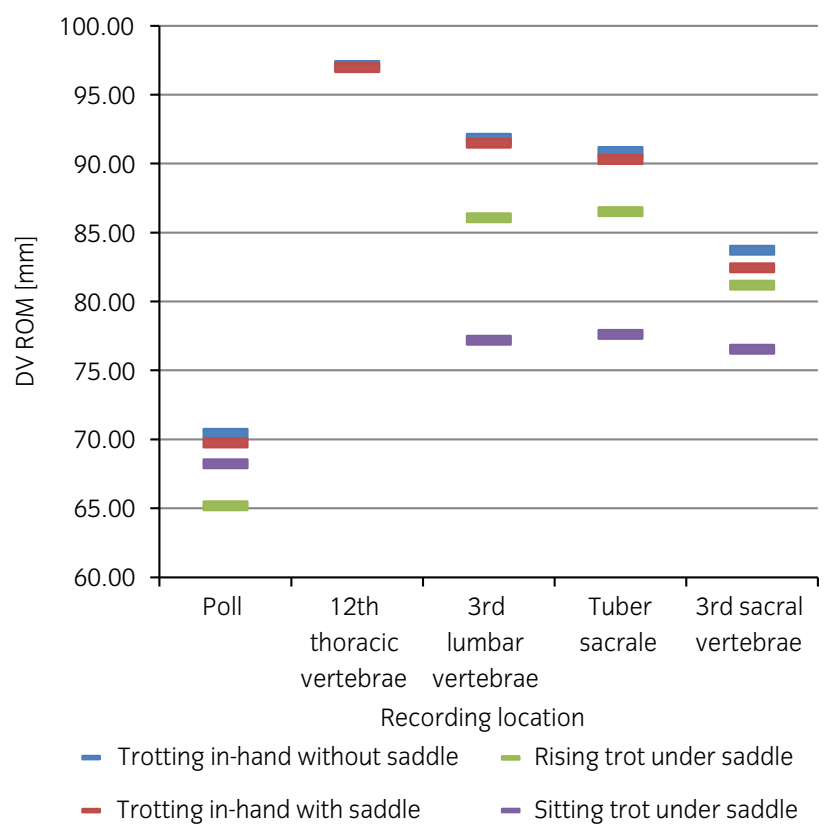

Fig 4: Mean dorsoventral range of motion (DV ROM; in $\mathrm{mm}$ ) of the vertebral column at the five locations along the spine shown in Figure 1 during trot under different conditions. Error bars represent 95\% lower and upper confidence limits of the mean. Note: measurements were not made from the 12th thoracic vertebral region when the horses were ridden.

no overall change to increased ROM at the trot whether a dead weight was used or the horse was ridden $[29,30]$. Our results suggest an overall reduction in vertebral DV ROM with a rider in place. This may be explained by an increased synergistic stabilising activity of the rectus abdominis and longissimus dorsi muscles restricting excessive movement during ridden trot and the associated increase in force [31,32].

Despite the use of an experienced dressage rider in the current study, ML movement of the head was significantly greater under saddle, demonstrating a horse-rider interaction [33,34]. Consistent with a limbback-head motion chain, in which each unit collaborates to maintain balance [24,35-40], we speculate that increased head and neck movement may compensate for a decrease in spinal movement further caudally. It is

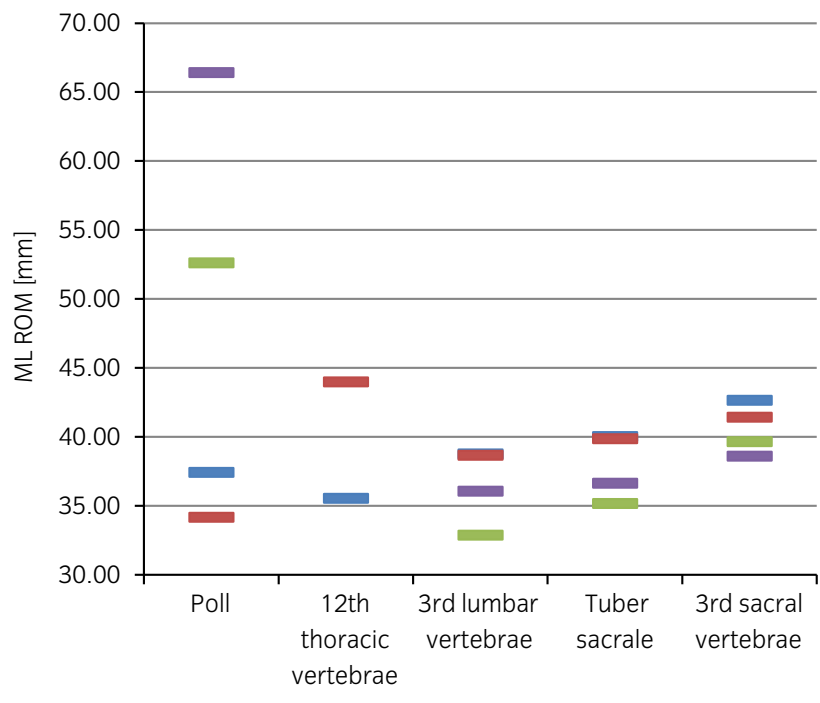

Recording location

- Trotting in-hand without saddle - Rising trot under saddle

- Trotting in-hand with saddle _ - Sitting trot under saddle

Fig 5: Mean mediolateral range of motion ( $\mathrm{ML} \mathrm{ROM}$; in $\mathrm{mm}$ ) of the vertebral column at the five locations along the spine shown in Fig 1 during trot under different conditions. Note: measurements were not made from the 12th thoracic vertebral region when the horses were ridden.

generally assumed that riding technique influences the loading of the horse's back, with rising trot being less demanding than sitting trot [41]. Assuming stabilising muscular activity counteracts the greater force peaks that occur during sitting trot, it is unsurprising that the current study recorded significantly lower ROM during sitting trot when compared with rising trot, consistent with previous studies [30].

We assessed parameters relevant to the biomechanics of the equine vertebral column in an attempt to explain the low variability and high repeatability in movement measures within the current population of Franches-Montagnes stallions. Age has been shown to influence equine back movement; for example, older horses have decreased flexion and extension at the transition between the thoracic and lumbar back at the trot [42]. Dividing horses into age groups for analysis was largely 
TABLE 3: Factors potentially influencing range of motion of the vertebral column and associations with different variability in the range of movement measurements in 27 Franches-Montagnes stallions and six general population horses analysed with the Modified Levene Equal Variance Test

\begin{tabular}{lllll}
\hline & & $\begin{array}{l}\text { Rump } \\
\text { length } \\
{[\mathrm{cm}]}\end{array}$ & $\begin{array}{l}\text { Height } \\
{[\mathrm{cm}]}\end{array}$ & $\begin{array}{l}\text { Rump } \\
\text { length:height } \\
\text { at withers }\end{array}$ \\
\hline Franches-Montagnes & & & & \\
Mean & 10.44 & 159.70 & 157.19 & 1.02 \\
Standard deviation (s.d.) & 4.80 & 4.01 & 2.80 & 0.02 \\
95\% LCL of the s.d. & 3.78 & 3.16 & 2.21 & 0.01 \\
95\% UCL of the s.d. & 6.58 & 5.49 & 3.84 & 0.02 \\
95\% LCL & 8.55 & 158.12 & 156.08 & 1.01 \\
95\% UCL & 12.34 & 161.29 & 158.29 & 1.02 \\
General population & & & & \\
Mean & 10.00 & 165.50 & 168.50 & 0.98 \\
Standard deviation & 3.10 & 11.38 & 6.66 & 0.04 \\
95\% LCL of the s.d. & 1.93 & 7.10 & 4.15 & 0.02 \\
95\% UCL of the s.d. & 7.60 & 27.91 & 16.32 & 0.09 \\
95\% LCL & 6.75 & 153.56 & 161.52 & 0.94 \\
95\% UCL & 13.25 & 177.44 & 175.48 & 1.02 \\
Modified Levene (P value) & $>0.05$ & $<0.001$ & $<0.001$ & $<0.03$ \\
\hline
\end{tabular}

$\mathrm{LCL}$ and $\mathrm{UCL}$, lower and upper confidence limits, respectively.

inconclusive (Supplementary Item S3). This apparent lack of a relationship between age and movement reduces the likelihood of age being an important factor in this population and this was confirmed by the results of the Modified Levene Equal Variance Test (Table 3).

Based on the heterogeneous nature of the general population it is not surprising that there was a higher variability in RL, HW and RL:HW in this group when compared with the Franches-Montagnes stallions. Anecdotal information on the role of conformation on back kinematics is abundant but scientific evidence is lacking. It has been suggested that increasing back length is associated with increased suppleness [43]. Interestingly, longer backs have been strongly correlated with less DV ROM at the lumbar spine, but a greater degree of lateral bending [44]. This observation was confirmed through the comparison of the lumbar region of dressage horses with show jumpers. Dressage horses had both longer lumbar backs and greater lateral movement [42]. These studies clearly show the influence of back length on ROM and therefore support our observation that low variability in this parameter may be associated with the low variability in ROM measured in the Franches-Montagnes stallions of this study.

The RL:HW ratio also influences back movement, and Johnston et al. [44] showed increased ML movement in horses with a rectangular shape. Franches-Montagnes horses are generally compact and square in shape. Attempts to determine differences in ROM by grouping the FranchesMontagnes stallions in this study according to their minimal differences in RL:HW failed to show any significant differences, highlighting their uniform conformation. Significantly greater variability in conformational factors (RL, HW, RL:HW) was shown in the general population horses when compared with Franches-Montagnes horses and may correlate with consistent movement through the vertebral column.

In order to define normal reference values, measurements should have low variability and high repeatability. Using IMU technology in a relatively uniform population (i.e. Franches-Montagnes stallions), we were able to establish normal values for ROM with a relatively narrow range. This was most evident when comparing with the range recorded in a random sample from the general population. These normal reference values may therefore serve as a benchmark for this breed and serve for comparison in cases suspected to have back pain. Due to variation in conformation, sex, utilisation and exercise history, extrapolating the current results to those of a general horse population is not possible. It remains to be seen if normal reference values can be determined for other breeds with a more or less uniform conformation.
The uniform nature of the population of horses and the conditions under which they were measured in this study is unquestionable. The presence of clinical back pain or lameness was excluded based on clinical examination. However, the acclimatisation to ridden exercise was variable as, although all horses were accustomed to ridden exercise, some were more routinely driven. The warm-up period prior to measurement was designed to compensate for this. Nonetheless variability in the 'condition' of the back within the population of Franches-Montagnes stallions and its influence on movement was not more closely accounted for. Further work is required to evaluate the feasibility of determining normal values for both individuals and populations of horses and to establish whether they are a useful basis for the diagnosis of clinical abnormalities. The IMUs used in this study are only validated for measuring total DV or ML excursion. Differentiating flexion, extension and left or right lateral excursion is likely to be of greater clinical relevance.

\section{Authors' declaration of interests}

No competing interests have been declared.

\section{Ethical animal research}

All the horses in the Franches-Montagnes group and two horses of the general population group are owned by the National Stud. Permission to examine them was granted by a representative of the stud. The remaining horses in the general population group are privately owned. Owners gave informed consent for their horses to be included.

\section{Source of funding}

This study was funded by the Department of Clinical Veterinary Medicine research fund, University of Berne, Switzerland.

\section{Acknowledgements}

The authors would like to thank Päivi Nussbaumer, Henrique Miguel Maya and the team of riders from the Swiss National Stud (Haras National Suisse) for their time and patience during data acquisition. Further technical support was provided by Alessandra Ramseyer, Tom Witte, Zeljko Kragic and the staff of the ISME, Avenches.

\section{Authorship}

All authors contributed to the study's conception and design and acquisition or analysis and interpretation of the data. While C. Heim drafted the article, the other authors revised it critically for important intellectual content. All authors have approved the final version of the manuscript.

\section{Manufacturers' addresses}

${ }^{a}$ Xsens Technologies B.V., P.O. Box 559, 7500 An Enschede, The Netherlands.

${ }^{\mathrm{b}}$ Animal Polster, Snoegg, Norway.

'The Mathworks, Natick, Massachusetts, USA.

${ }^{\mathrm{d}}$ NCSS, LLC., Kaysville, Utah, USA.

\section{References}

1. McCracken, M.J., Kramer, J., Keegan, K.G., Lopes, M., Wilson, D.A., Reed, S.K., LaCarrubba, A. and Rasch, M. (2012) Comparison of an inertial sensor system of lameness quantification with subjective lameness evaluation. Equine Vet. J. 44, 652-656.

2. Church, E.E., Walker, A.M., Wilson, A.M. and Pfau, T. (2009) Evaluation of discriminant analysis based on dorsoventral symmetry indices to quantify hindlimb lameness during over ground locomotion in the horse. Equine vet. J. 41, 304-308. 
3. Pfau, T., Robilliard, J.J., Weller, R., Jespers, K., Eliashar, E. and Wilson, A.M. (2007) Assessment of mild hindlimb lameness during over ground locomotion using linear discriminant analysis of inertial sensor data. Equine Vet. J. 39, 407-413.

4. Parkes, R.S., Weller, R., Groth, A.M., May, S. and Pfau, T. (2009) Evidence of the development of 'domain-restricted' expertise in the recognition of asymmetric motion characteristics of hindlimb lameness in the horse. Equine Vet. J. 41, 112-117.

5. Warner, S.M., Koch, T.O. and Pfau, T. (2010) Inertial sensors for assessment of back movement in horses during locomotion over ground. J. Equine Vet. 42, Suppl. 38, 417-424.

6. Buchner, H.H., Savelberg, H.H., Schamhardt, H.C. and Barneveld, A. (1996) Head and trunk movement adaptations in horses with experimentally induced fore- or hindlimb lameness. Equine Vet. J. 28, 71-76.

7. Pfau, T., Witte, T.H. and Wilson, A.M. (2005) A method for deriving displacement data during cyclical movement using an inertial sensor. J. Exp. Biol. 208, 2503-2514

8. Pfau, T., Witte, T.H. and Wilson, A.M. (2006) Centre of mass movement and mechanical energy fluctuation during gallop locomotion in the Thoroughbred racehorse. J. Exp. Biol. 209, 3742-3757.

9. Starke, S.D., Witte, T.H., May, S.A. and Pfau, T. (2012) Accuracy and precision of hind limb foot contact timings of horses determined using a pelvis-mounted inertial measurement unit. J. Biomech. 45, 1522-1528.

10. Hoyt, D.F., Wickler, S.J., Dutto, D.J., Catterfeld, G.E. and Johnsen, D. (2006) What are the relations between mechanics, gait parameters, and energetics in terrestrial locomotion? J. Exp. Zool. A. Comp. Exp. Biol. 305, 912-922.

11. Wickler, S.J., Hoyt, D.F., Cogger, E.A. and McGuire, R. (2002) The cost of transport in an extended trot. Equine Vet. J. 34, Suppl. 34, 126-130

12. Starke, S.D., Raistrick, K.J., May, S.A. and Pfau, T. (2013) The effect of trotting speed on the evaluation of subtle lameness in horses. Vet. J. 197, 245-252.

13. Jeffcott, L.B. and Dalin, G. (1980) Natural rigidity of the horse's backbone. Equine Vet. J. 12, 101-108.

14. Townsend, H.G. and Leach, D.H. (1984) Relationship between intervertebral joint morphology and mobility in the equine thoracolumbar spine. Equine Vet. J. 16, 461-465

15. Denoix, J.M. (1999) Spinal biomechanics and functional anatomy. Vet. Clin. N. Am.: Equine Pract. 15: 27-60.

16. Faber, M., Johnston, C., Schamhardt, H., van Weeren, R., Roepstorff, L. and Barneveld, A. (2001) Basic three-dimensional kinematics of the vertebral column of horses trotting on a treadmill. Am. J. Vet. Res. 62, 757-764.

17. de Cocq, P., van Weeren, P.R. and Back, W. (2004) Effects of girth, saddle and weight on movements of the horse. Equine Vet. J. 36, 758763.

18. Pfau, T., Stubbs, N.C., Kaiser, L.J., Brown, L.E. and Clayton, H.M. (2012) Effect of trotting speed and circle radius on movement symmetry in horses during lunging on a soft surface. Am. J. Vet. Res. 73, 18901899.

19. Brocklehurst, C., Weller, R. and Pfau, T. (2014) Effect of turn direction on body lean angle in the horse in trot and canter. Vet. J. 199, 258-262.

20. Goff, L., Van Weeren, P.R., Jeffcott, L., Condie, P. and McGowan, C. (2010) Quantification of equine sacral and iliac motion during gait: a comparison between motion capture with skin-mounted and bone-fixated sensors. Equine Vet. J. 42, Suppl. 38, 468-474

21. Taylor, W.R., Ehrig, R.M., Duda, G.N., Schell, H., Seebeck, P. and Heller, M.O. (2005) On the influence of soft tissue coverage in the determination of bone kinematics using skin markers. J. Orthop. Res. 23, 726-734.

22. van den Bogert, A.J., van Weeren, P.R. and Schamhardt, H.C. (1990) Correction for skin displacement errors in movement analysis of the horse. J. Biomech. 23, 97-101.

23. van Weeren, P.R. and Barneveld, A. (1986) A technique to quantify skin displacement in the walking horse. J. Biomech. 19, 879-883.

24. Gomez Alvarez, C.B., Rhodin, M., Bystrom, A., Back, W. and van Weeren, P.R. (2009) Back kinematics of healthy trotting horses during treadmill versus over ground locomotion. Equine Vet. J. 41, 297-300.

25. Meyer, H. (1999) Studies of the effect of the rider's weight and hand actions on the motion of the horse. Tierarztl. Umsch. 54, 6.
26. Preuschoft, H., Falaturi, P. and Lesch, C. (1995) The influence of riders on their horses. Tierarztl. Umsch. 50, 11.

27. Clayton, H.M., Lanovaz, J.L., Schamhardt, H.C. and van Wessum, R. (1999) The effects of a rider's mass on ground reaction forces and fetlock kinematics at the trot. Equine Vet. J. 31, Suppl. 30, 218-221.

28. Slijper, E.J. (1946) Comparative biological-anatomical investigations on the vertebral column and spinal musculature of mammals. Proc. K. Ned. Akad. Wet. Verh (Tweede sectie) 47: 1-128.

29. Valentin, S., Davis, R., Wilson, A. and Pfau, T. (2010) The effect of loading on the equine spine - A preliminary study. Wien. Tierarztl. Monatsschr. 97, 7.

30. De Cocq, P., Prinsen, H., Springer, N.C., van Weeren, P.R., Schreuder, M., Muller, M. and van Leeuwen, J.L. (2009) The effect of rising and sitting trot on back movements and head-neck position of the horse. Equine vet. J. 41, 423-427.

31. Rooney, J.R. (1982) The horse's back: biomechanics of lameness. Equine Pract. 4, 11.

32. Robert, C., Audigie, F., Valette, J.P., Pourcelot, P. and Denoix, J.M. (2001) Effects of treadmill speed on the mechanics of the back in the trotting saddlehorse. Equine Vet. J. 33, Suppl. 33, 154-159.

33. Clayton, H.M., Larson, B., Kaiser, L.J. and Lavagnino, M. (2011) Length and elasticity of side reins affect rein tension at trot. Vet. J. 188, 291-294.

34. Greve, L. and Dyson, S. (2013) The horse-saddle-rider interaction. Vet. J. 195, 275-281.

35. Gomez Alvarez, C.B. (2008) The back of the horse: a bridge between the extremities, but functionally not enough understood. Tijdschr. Diergeneeskd. 133, 804-806.

36. Gomez Alvarez, C.B., Bobbert, M.F., Lamers, L., Johnston, C., Back, W. and van Weeren, P.R. (2008) The effect of induced hindlimb lameness on thoracolumbar kinematics during treadmill locomotion. Equine Vet. J. 40, 147-152.

37. Gomez Alvarez, C.B., L'Ami, J.J., Moffat, D., Back, W. and van Weeren, P.R. (2008) Effect of chiropractic manipulations on the kinematics of back and limbs in horses with clinically diagnosed back problems. Equine Vet. J. 40, 153-159.

38. Gomez Alvarez, C.B., Rhodin, M., Bobber, M.F., Meyer, H., Weishaupt, M.A., Johnston, C. and Van Weeren, P.R. (2006) The effect of head and neck position on the thoracolumbar kinematics in the unridden horse. Equine Vet. J. 38, Suppl. 36, 445-451.

39. Gomez Alvarez, C.B., Wennerstrand, J., Bobbert, M.F., Lamers, L., Johnston, C., Back, W. and van Weeren, P.R. (2007) The effect of induced forelimb lameness on thoracolumbar kinematics during treadmill locomotion. Equine Vet. J. 39, 197-201.

40. Rhodin, M., Gomez Alvarez, C.B., Bystrom, A., Johnston, C., van Weeren, P.R., Roepstorff, L. and Weishaupt, M.A. (2009) The effect of different head and neck positions on the caudal back and hindlimb kinematics in the elite dressage horse at trot. Equine Vet. J. 41, 274-279.

41. de Cocq, P., Duncker, A.M., Clayton, H.M., Bobbert, M.F., Muller, M. and van Leeuwen, J.L. (2010) Vertical forces on the horse's back in sitting and rising trot. J. Biomech. 43, 627-631.

42. Johnston, C., Holm, K.R., Erichsen, C., Eksell, P. and Drevemo, S. (2004) Kinematic evaluation of the back in fully functioning riding horses. Equine Vet. J. 36, 495-498.

43. Jeffcott, L.B. (1999) Back problems. Historical perspective and clinical indications. Vet. Clin. N. Am.: Equine Pract. 15: 1-12.

44. Johnston, C., Holmt, K., Faber, M., Erichsen, C., Eksell, P. and Drevemo, S. (2002) Effect of conformational aspects on the movement of the equine back. Equine Vet. J. 34, Suppl. 34, 314-318.

\section{Supporting Information}

Additional Supporting Information may be found in the online version of this article at the publisher's website:

Supplementary Item 1. Dorsoventral (DV) and mediolateral (ML) range of motion of the vertebral column measurements of 27 Franches-Montagnes stallions. Mean, standard deviation (s.d.) and 95\% lower (LCL) and upper confidence limits (UCL) in five spinal locations (shown in Fig 1) under various exercise conditions. 
Supplementary Item 2. Dorsoventral (DV) and mediolateral (ML) range of motion of the vertebral column measurements of 6 general population horses. Mean, standard deviation (s.d.) and 95\% lower (LCL) and upper confidence limits (UCL) in 5 spinal locations (shown in Fig 1) under various exercise conditions.

Supplementary Item 3. Repeated measures ANOVA examining the impact of age on range of movement of the vertebral column in 27 Franches-Montagnes stallions in various exercise conditions by dividing them into two age groups (DV, dorsoventral; ML, mediolateral).
Supplementary Item 4. Repeated measures ANOVA examining the impact of age on range of movement of the vertebral column in 27 Franches-Montagnes stallions in various exercise conditions by dividing them into three age groups (DV, dorsoventral; ML, mediolateral).

Supplementary Item 5. Repeated measures ANOVA examining the impact of age on range of movement of the vertebral column in 27 Franches-Montagnes stallions in various exercise conditions by dividing them into four age groups (DV, dorsoventral; ML, mediolateral). 